\title{
An Inclusive Philosophy of Mathematics
}

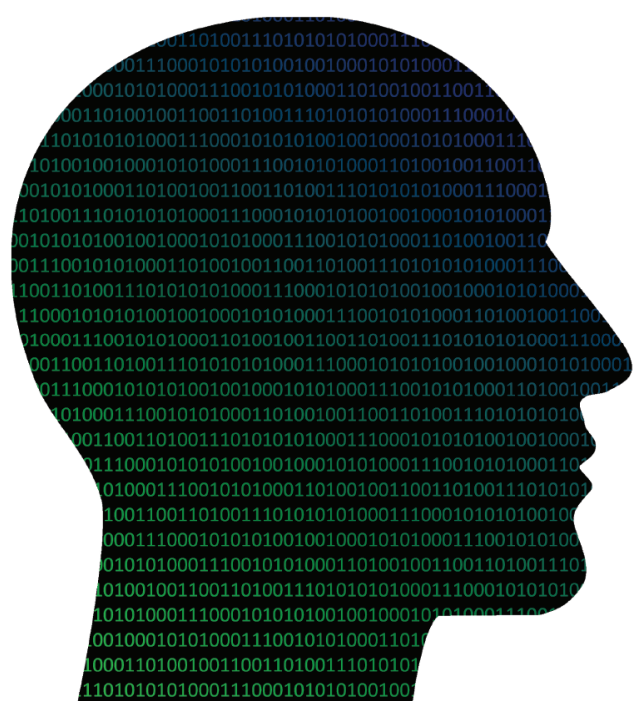

John Hosack

The Bulletin of the AMS recently published expositions of two varieties of mathematics: constructive mathematics in [Bau16] and univalent foundations in [Gra18]. Standard mathematics (which is what most mathematicians learn, teach, and use), constructive mathematics, and univalent foundations have incompatible logical or mathematical foundations. How should a mathematician put these alternative varieties of mathematics into a context that takes into account the interest shown by their publication in a leading journal? Some of these, and other, contradictory varieties of mathematics have long been studied by respected mathematicians for reasons including tradition, philosophy, or applications. The purpose of this paper is not to adjudicate between varieties or recommend one for study. Rather, the question here is how a mathematician working within one variety should view another, contradictory variety; in particular should it be rejected as false? In this paper we justify an inclusive attitude that does not reject

John Hosack is a retired mathematician. His email address is hosack@ alumni . caltech.edu .

For permission to reprint this article, please contact: reprint-permission aams.org.

DOI: https://dx.doi.org/10.1090/noti1942 other varieties as false and consider deductivism within a variety as a consequence of this inclusive attitude. To do this a suitable context must have a broad view of mathematics to include the fact of these varieties. The objective of this paper is to provide a suitable perspective in the form of a new philosophy of mathematics: deductive pluralism.

This paper will use the term "variety," analogously to [Sha14], but herein a variety will be a maximal set of results (including definitions, theorems, and examples) following from logical and mathematical foundations. For example, standard mathematics is the mathematics that most mathematicians learn and use and has First Order Predicate Calculus (FOPC) as the logical foundation and Zermelo-Fraenkel set theory with the Axiom of Choice (ZFC) as the mathematical foundation. This paper proposes that a philosophy of mathematics should be inclusive of the varieties of mathematics. As used here, inclusive means that a variety should not be viewed from a philosophical perspective as false or excluded from consideration; it does not mean that an individual should not prefer one variety over others. Also, as discussed below, an inclusive philosophy should also take into account the problematic question of the existence of abstract objects (those that do not exist in space-time) and the relative nature of consistency results. 
An outline of deductive pluralism and the argument favoring it is as follows: a philosophy of mathematics should be inclusive of existing mathematics; there are existing varieties of mathematics with incompatible logical or mathematical foundations; the criterion of inclusiveness and the fact of incompatible varieties implies a pluralistic philosophy; this in turn requires a deductivist approach (which as a motto asserts that mathematics studies "what follows from what") within each variety, since if the foundations were viewed as false, then the variety would likely be rejected, thus deductive pluralism. In the following sections this paper will: clarify the concept of a variety with several examples; argue in favor of the criterion of inclusiveness and the need for a deductive approach; discuss some of the advantages of deductive pluralism; and finally put deductive pluralism into the context of the philosophy of mathematics, including references to other work on deductivism, pluralism, and deductive pluralism.

\section{Examples of Varieties of Mathematics}

This section gives examples of varieties of mathematics with incompatible logical or mathematical foundations with the information needed for subsequent discussion and indicates some of the motivations that lead to these varieties. There are many other varieties, such as category theory as a foundation, various forms of finitism, and even inconsistent mathematics, but those discussed here should be sufficient for our purposes.

While working within a variety, the logic of that variety is applied to the previous results to deduce theorems, to make definitions, or to construct examples. In this paper the discussion of varieties has the purpose of demonstrating incompatible logical or mathematical foundations. Thus, the pluralism advocated is descriptive of the existing situation rather than prescriptive.

Recall that a system is inconsistent if for some proposition $\mathrm{P}$, both $\mathrm{P}$ and its negation $\neg \mathrm{P}$ can be derived and is consistent if not inconsistent. If a system is inconsistent, then in principle this can be proven by systematically listing all proofs. However, if the system is consistent, then this process will not terminate. From Gödel's second incompleteness theorem most consistent mathematical systems of interest cannot prove their own consistency. Thus for these systems assertions of relative consistency are made rather than assertions of consistency (where system A is consistent relative to system $B$ if the consistency of B implies the consistency of A). As an example consider Peano Arithmetic (PA), the axiomatization of ordinary arithmetic. PA is either consistent or not, but since it satisfies the conditions of Gödel's theorem, if it is consistent, then it cannot prove its own consistency. However, it can be embedded in set theory, specifically in its standard axiomatization ZFC. Thus, PA is consistent relative to ZFC. Usually mathematicians (implicitly) assume that the variety they are working in, such as PA or ZFC, is consistent.
In parallel with relative consistency, existence of mathematical objects should be viewed as relative to a variety. More explicitly, a concept can be asserted to exist in a variety if it can be obtained from the foundations by a sequence of results: definitions, theorems, and examples. For example, the concept of a group can be defined using the logic and foundations of ZFC. Examples of groups can then be constructed using the axioms to show that the concept is not vacuous.

The first example of a variety of mathematics will be standard mathematics, which is based on FOPC as the logic and on ZFC as the mathematical component of the foundation (or just ZFC if the logic FOPC is understood). Since standard mathematics is so dominant and extensive most other varieties of mathematics, including those discussed below, are careful to include many of the same or similar theories and results as standard mathematics. As noted above, PA can be considered as a variety of mathematics or as a theory within ZFC.

The next example is constructive mathematics (see [Bau16]), of which there are several versions. The version considered here, sometimes referred to as BISH, was developed from the work of Errett Bishop and is viewed as a basis for several versions of constructive mathematics. BISH is an example of a variety of mathematics in which the mathematical assertions and logic have both rules and interpretations different from standard mathematics. There are two requirements: the existence of a mathematical object can be asserted only if there is an algorithmic construction for the object, and the truth of a proposition can be asserted only when it can be proven. For example, if $P$ and $Q$ are propositions, then the disjunction $P \vee Q$ can be asserted only when there is a proof for $P$ or for $Q$. However, if we set $Q=\neg P$, then we can assert $P \vee \neg P$ only if we can prove either $P$ or $\neg P$, which is not always possible. Thus, constructive mathematics does not accept the Law of Excluded Middle (LEM), which asserts that $P \vee \neg P$ always holds. The logical foundation is intuitionistic logic, which is FOPC without LEM, and the mathematical foundations can be formalized as intuitionistic set theory. As an example of existence consider the comb (or Dirichlet) function, which is defined on the unit interval so that it is 1 on the rational numbers and 0 on the irrational numbers in the interval and which is a primary example of a bounded function on a bounded interval that is not Riemann integrable but is Lebesgue integrable. The existence of the comb function can be asserted in standard mathematics but not in BISH.

Standard mathematics is a proper extension of BISH, so that all theorems of BISH are also theorems of standard mathematics, but not conversely. Thus, BISH is consistent relative to ZFC. As discussed above, standard mathematics can be viewed as an extension of PA, just as standard mathematics can be viewed as an extension of BISH. However, the situations differ: standard mathematics does not contradict the motivations or logic of PA but does contradict BISH, 
since, for example, standard mathematics accepts LEM, but BISH rejects LEM.

Just as PA is the arithmetic theory in standard mathematics, Heyting Arithmetic (HA) is the arithmetic theory in constructive mathematics. PA and HA have the same mathematical axioms, but HA uses intuitionistic logic (FOPC without LEM) rather than FOPC. Since HA has the same axioms and a weaker logic, any theorem in HA is a theorem in PA, but not conversely. For example, in HA a statement of the form $\neg \forall n \phi(n)$ does not necessarily imply that $\exists n \neg \phi(n)$, since in HA existence can be asserted only if there is a construction. Finally, Gödel proved in 1933 that PA is consistent if and only if HA is consistent.

Tarski-Grothendieck set theory (TG) is a proper extension of ZFC with FOPC as the logic. A motivation is to provide a basis for the work of Grothendieck and others in algebraic geometry. TG adds an axiom U to ZFC stating that every set is an element of a Grothendieck universe, where a Grothendieck universe is a set defined so that it is closed under the usual set operations such as the power set. Since the axioms of ZFC are a subset of the axioms of TG, ZFC is consistent if TG is consistent, and a Grothendieck universe acts as an interpretation of ZFC within TG.

The final example is the univalent foundations program (see [Gra18]), a recent development given impetus by Voevodsky and with a motivation stated in the subtitle "A Personal Mission to Develop Computer Proof Verification to Avoid Mathematical Mistakes" of the paper by Voevodsky [Voe14, p. 8]. It is an example of a variety of mathematics not based on set theory. Instead, it has as its basis an extension of the predicative, intuitionistic Martin-Löf type theory with additional axioms such as univalence. Just as standard set theory assumes the existence of the empty set and has axioms that assert the existence of new sets given existing sets (e.g., unions), univalent foundations assumes the needed types, such as the natural number type. The logic is intuitionistic, and in this approach there are several primitive concepts, including type, identity of types, function types, and ordered pairs. However, unlike constructive mathematics assumptions outside intuitionistic logic, such as LEM, are introduced as hypotheses to theorems when needed. The univalence axiom implies that isomorphic structures can be identified. Identifying structures up to isomorphism is common in standard mathematics; e.g., the von Neumann and other interpretations of the natural numbers are isomorphic in standard set theory and thus can be considered identical as a type. However, in standard mathematics isomorphic objects are not necessarily identified. For example, the singleton sets $\{0\}$ and $\{1\}$ are isomorphic as sets (and by a unique isomorphism), but if they are identified, then by extensionality the elements would be the same, and so as a consequence $0=1$. Thus, univalent foundations are incompatible with standard set theory. However, much of univalent foundations, including the univalence axiom, has been shown to be consistent relative to ZFC.

\section{Incompatible Varieties of Mathematics and Deductive Pluralism}

The previous section briefly examined several varieties of mathematics: ZFC; TG, an extension of ZFC; constructive, a restriction of ZFC; and univalent foundations, not based on set theory. If a philosophy of mathematics is to be inclusive of mathematical practice, then it must accommodate these varieties, which have different logical assumptions (e.g., FOPC or intuitionistic), different set-theoretic foundations (e.g., ZFC or TG), or foundations not using set theory (e.g., univalent foundations). As a consequence objects may exist in one variety of mathematics but not in another variety (e.g., a Grothendieck universe exists in TG but not in standard mathematics; the comb function exists in standard mathematics but not in BISH), or two varieties may have the same logic but different axioms (e.g., TG and ZFC) or different logics but the same axioms (e.g., PA and HA). Thus, the discussions of the above varieties show that no single logical or mathematical foundation is feasible.

The preceding section demonstrated the existence of incompatible varieties of mathematics. Now the criterion of inclusiveness is considered. Some mathematicians assume the absolute truth of the foundations of a particular variety for reasons including tradition, philosophy, or applications. These assumptions may be unfalsifiable, especially since there is no general agreement on the criteria for the existence of abstract objects, as will be discussed in more detail in the next section. So, the criterion of inclusiveness must be an assumption. However, it is an assumption with strong justifications. Although when foundations are considered some may regard a particular variety as uniquely correct or worthy of study, from a philosophical point of view an inclusive perspective should be maintained. Otherwise, there are two problems. The first is that a philosophy that rejects and excludes from consideration some varieties of mathematics will be a philosophy of only a part of mathematics, e.g., of constructive mathematics, which might be fine as far as it goes, but it would not be a philosophy of mathematics as a whole. The second problem is that there will be no generally acceptable and principled criteria for what is to be excluded. The lack of such acceptable and principled criteria is shown by the support given by respected mathematicians such as Bishop to constructive mathematics, Grothendieck to TG, and Voevodsky to univalent foundations. Based on these considerations an inclusive and thus pluralistic attitude should be maintained.

A pluralistic philosophy of mathematics should be combined with a deductive approach within a variety. By this is meant that a mathematical assertion implicitly or explicitly states that a conclusion follows from assumptions, ultimately from the logical and mathematical foundations after a long development of intermediate results 
(which include definitions, examples, and theorems). In deductivism (also referred to by some as if-thenism) the foundational assumptions are not considered true in some absolute sense but as a basis for work within the variety. For example, foundational assumptions in set theory may include the existence of an empty set and the existence of an infinite set. Additional set-theoretic assumptions will assert that if some sets exist, then others exist, such as their union. A deductivist approach will not assert that the empty set exists or that an infinite set exists in some absolute sense (a problematic question for abstract objects) but rather will ask what conclusions can be drawn from such assumptions. Thus, assertions of truth, existence, or consistency become relative rather than absolute, which is compatible with the fact that mathematical theorems about existence and consistency are, implicitly or explicitly, relative to the variety.

If, contrary to deductivism, some foundational assumptions of a variety are considered as true in some absolute sense, then other, contradictory assumptions would be viewed as false. A philosophy that viewed foundational assumptions of a variety as false would reject that variety, violating the inclusiveness criterion. Thus, results within a variety must be viewed as holding or not relative to the foundational assumptions of that variety. So, rather than assigning truth or falsity in some absolute sense to foundational assumptions, the question becomes whether the results are correctly deduced from or are consistent with the assumptions: a deductive stance.

\section{Some Advantages of Deductive Pluralism}

One of the philosophical advantages of any version of deductivism is the elimination of ontological problems, since no variety is considered as true in some absolute sense, and the basic statements are assertions that the assumptions (ultimately the foundations) imply the conclusions. Thus, there are no problematic questions about the existence of abstract objects.

Any attempt to go beyond deductivism requires confronting the problematic question of the existence of abstract objects. Within philosophy there is no agreement on whether or not abstract objects exist, on the conditions for their existence, or even if the question is meaningful. One of the clearest approaches to abstract objects within mathematics is that of Hilbert, who equated existence of such objects with consistency in his 1900 address introducing the Hilbert Problems. He stated:

If contradictory attributes be assigned to a concept, I say that mathematically the concept does not exist....But if it can be proved that the attributes assigned to the concept can never lead to a contradiction by the application of a finite number of logical inferences, I say that the mathematical existence of the concept...is thereby proved.
One problem is that, as noted above, consistency results have become relative. A related problem is the multiplicity of contradictory but relatively consistent axiom systems, so that if truth is identified with consistency, then there are contradictory true statements. For example, consider the Continuum Hypothesis $(\mathrm{CH})$, which states that any infinite subset of the reals must have the same cardinality as (be equinumerous with) either the reals or the natural numbers. If ZFC is assumed to be a consistent basis for mathematics, then both $\mathrm{ZFC}+\mathrm{CH}$ and $\mathrm{ZFC}+\neg \mathrm{CH}$ are also consistent (by the work of Gödel and Cohen). So, using consistency as the criterion for existence, if the existential assertions of ZFC hold, then so do the contradictory assertions of $\mathrm{CH}$ and $\neg \mathrm{CH}$. (Notice the difference between this contradiction within one theory, the real numbers, and two distinct theories with incompatible assumptions that have examples within standard mathematics, e.g., Euclidean and non-Euclidean geometry.) Thus, the initially attractive idea of identifying mathematical existence with consistency runs aground on the results since the 1900 formulation.

In deductive pluralism mathematical statements take the form of assertions that the assumptions, ultimately the foundations, imply the conclusions. With this approach the assertions (i.e., implications) are also objectively true in that mathematicians favoring different varieties of mathematics can agree that within another variety given the assumptions, the logic to be used, and a deduction correctly using this logic, the conclusion follows (possibly verified using computer proof assistants such as Mizar for TG and Coq for univalent foundations).

\section{Deductive Pluralism in the Philosophy of Mathematics}

Deductive pluralism has two components: inclusive pluralism and deductivism within a variety. There has been some discussion and acceptance of inclusive pluralism in related subjects, such as logic [Sha14]; however there have been no efforts before deductive pluralism to consider that inclusive pluralism in mathematics needs deductivism within a variety. Deductivism has been considered for more than a century but (implicitly) within the context of a single variety. For example, Bertrand Russell wrote in 1903 that "Pure Mathematics is the class of all propositions of the form 'p implies q,' [Rus03, p. 3]. The consideration of deductivism over the years has sometimes led to the assertion that deductivism has significant advantages but that there are also serious objections to deductivism. Some objections depend on the (implicit) assumption of only one variety of mathematics and so do not apply to deductive pluralism. Space does not allow a substantial discussion of the objections to deductivism that have been made, so only the most commonly made objection will be briefly considered here. (For a more complete discussion of the objections to deductivism and answers to these objections, see [Hos19].) 
Of the objections that might apply to deductive pluralism the most common is the question of how deductivism relates to applications; e.g., see Maddy [Mad89]. These objections are discussed in detail in [Hos19], but briefly there are several points to note. First, incompatible varieties, such as constructive and standard mathematics, can be applied, so the problem of incompatible assumptions of different varieties is not eliminated by considering applications. Second, since the assumptions of a variety are not arbitrary but reflect centuries of work in mathematics, science, and philosophy, it is not surprising that the deductions can be relevant to areas outside mathematics. Finally, within a large and flexible variety there may be mathematics that is applicable to incompatible scientific theories, such as Newtonian physics versus general relativity or to discrete versus continuous systems.

In conclusion, given the incompatible varieties of mathematics, the natural condition that a philosophy of mathematics be inclusive leads to deductive pluralism. The advantages of this philosophy include the elimination of problematic questions about the existence of abstracta and the objectivity of mathematical assertions when considered as deductions. The advantages are not weakened by the objections to deductivism briefly mentioned here and discussed in detail in [Hos19].

\section{References}

[Bau16] Bauer A, Five stages of accepting constructive mathematics, Bull. Amer. Math. Soc. (N.S.) 54 (2017), 481-498. MR3662915

[Gra18] Grayson D, An introduction to univalent foundations for mathematicians, Bull. Amer. Math. Soc. (N.S.) 55 (2018), 427-450. MR3854072

[Hil02] Hilbert D, Mathematical problems, Bull. Amer. Math. Soc. 8 (1902), no. 10, 437-479. MR1557926

[Hos19] Hosack J, Deductive pluralism (2019), available at phi1sci-archive.pitt.edu/15805/.

[Mad89] Maddy P, The roots of contemporary platonism, $J$. Symbolic Logic 54 (1989), no. 4, 1121-1144.MR1026593

[Rus03] Russell B, Principles of mathematics, Cambridge University Press, Cambridge, 1903.

[Sha14] Shapiro S, Varieties of logic, Oxford University Press, Oxford, 2014. MR3241589

[Voe14] Voevodsky V, The origins and motivations of univalent foundations, The Institute Letter (2014), 8-9, available at https://www.ias.edu/ideas/2014/voevodsky -origins.

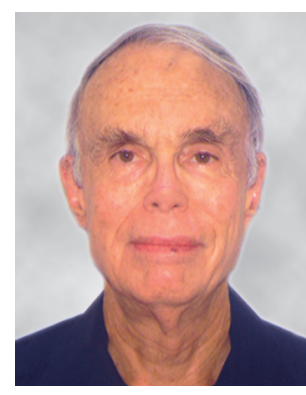

John Hosack

\section{Credits}

Opener image is by Tumisu from Pixabay.

Author photo is courtesy of the author.

\section{9-2020 MEMBERSHIP}

IAS

\section{MEMBERSHIPS}

THE IAS SCHOOL OF MATHEMATICS welcomes applications from mathematicians and theoretical computer scientists at all career levels, and strongly encourages applications from women, minorities, and mid-career scientists (5-15 years from Ph.D.). Competitive salaries, on-campus housing, and other resources are available for periods of $4-11$ months for researchers in all mathematical subject areas. The School supports approximately 40 post-docs per year.

In 2020-2021, there will be a special-year program, GEOMETRIC AND MODULAR REPRESENTATION THEORY, led by Geordie Williamson of the University of Sydney; however, Membership will not be limited to mathematicians in this field. To apply, submit your application at mathjobs. For more info, please visit: math.ias.edu

\section{MID-CAREER Are you 5-15 years from your Ph.D.? Ask us about funding!}

\section{PROGRAMS}

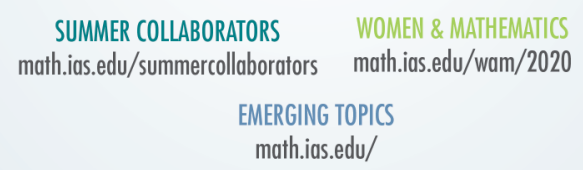

SUMMER COLLABORATORS WOMEN \& MATHEMATICS math.ias.edu/summercollaborators math.ias.edu/wam/2020

EMERGING TOPICS math.ias.edu/ 